\title{
In Vivo Imaging of Drosophila Larval Neuromuscular Junctions to Study Synapse Assembly
}

\author{
Till F.M. Andlauer and Stephan J. Sigrist
}

In the past decade, a significant number of proteins involved in the developmental assembly and maturation of synapses have been identified. However, detailed knowledge of the molecular processes underlying developmental synapse assembly is still sparse. Here, we discuss an approach that makes extended in vivo imaging of selected proteins in live Drosophila larvae feasible at a single-synapse resolution. The intact larvae are anesthetized and neuromuscular junctions (NMJs) are noninvasively imaged with confocal microscopy. This method allows for both protein trafficking and protein turnover kinetics to be studied at various points in time during the development of an animal. These data contribute to our understanding of synaptic assembly under in vivo conditions.

Chemical synapses are specialized for rapid directional signaling and are among the most elaborate signaling machines of cells. Recent work suggests that in vivo synaptogenesis is a long and intricate process involving multiple interrelated steps with reciprocal induction, as well as independent assembly of pre- and postsynaptic structures. Although data acquired in neuron cultures show that synapses can assemble quickly (Garner et al. 2006), other studies analyzing mammalian tissue samples suggest that this process takes $\sim 1 \mathrm{~d}$ (Nägerl et al. 2007). Thus, it is conceivable that regulation of synapse formation differs between in vitro and in vivo models. At present, a lack of knowledge concerning the detailed spatiotemporal sequence of in vivo synaptic assembly remains a barrier to a comprehensive understanding of the development of synaptic circuits. This issue could be overcome by extended molecular intravital imaging of individual synaptic proteins at identified sets of synapses.

The Drosophila larval NMJ is a leading model system to study synapses and their molecular foundation. It is especially well suited for the analysis of synaptic assembly for several reasons: Larval muscles are built and arranged in a stereotypical, repetitive manner, as are the motor neurons innervating them (Crossley 1978; Landgraf and Thor 2006). Therefore, the same individual NMJs can be easily identified among different individual larvae as well as within one particular larva at several points in time. It is thus possible to follow a defined NMJ and its population of synapses over time. The basic geometry of an individual NMJ is established during embryonic stages. With time, however, NMJs grow and expand continuously, constantly gaining additional boutons and individual synapses. Thus, at Drosophila NMJs, assembly of newly forming synapses can be investigated throughout all larval stages over relatively short periods of time.

The study of Drosophila larval NMJs is attractive because of the availability of many methodological approaches (Prokop and Meinertzhagen 2006). The ultrastructure of the synapses is thoroughly

Adapted from Drosophila Neurobiology (ed. Zhang et al.). CSHL Press, Cold Spring Harbor, NY, USA, 2010.

(C) 2012 Cold Spring Harbor Laboratory Press

Cite this article as Cold Spring Harb Protoc; 2012; doi:10.1101/pdb.top068577 
described and they are easily accessible for electrophysiological methods, which have been applied in numerous studies. In addition, the GAL4-UAS system (Brand and Perrimon 1993) allows for the restriction of expression of transgenic constructs to either the presynaptic (motor neuron) or postsynaptic (muscle) cell, allowing for the functional definition of the place of action of synaptic proteins by rescue experiments. Moreover, Drosophila NMJs show substantial, genetically evoked, and experience-mediated structural and functional plasticity (Zhong et al. 1992; Sigrist et al. 2003) and have significant similarities to glutamatergic excitatory synapses of the vertebrate central nervous system (Featherstone and Broadie 2000). Many of their key molecules are phylogenetically highly conserved (Keshishian et al. 1996).

An NMJ appears like beads on a string with each bead representing a bouton, which can be as large as $5 \mu \mathrm{m}$ in diameter (Broadie and Richmond 2002) (Fig. 1A-C). Different types of motor neuron boutons have distinct physiological as well as morphological properties and are categorized into three classes. Type-I boutons form glutamatergic synapses; they show a stereotypic morphology and can be further subdivided into type-Ib and -Is boutons. The superficial muscles 26 (VA1) and 27 (VA2) are well suited for intravital imaging (Fig. 1A). They have only type-Ib boutons, which are larger boutons that mainly contain clear synaptic vesicles (SVs; Landgraf et al. 2003; Prokop 2006).

Throughout larval development, the number of synaptic boutons at NMJs and individual synapses decorating these boutons increases significantly on a timescale of hours (Schuster et al. 1996; Collins

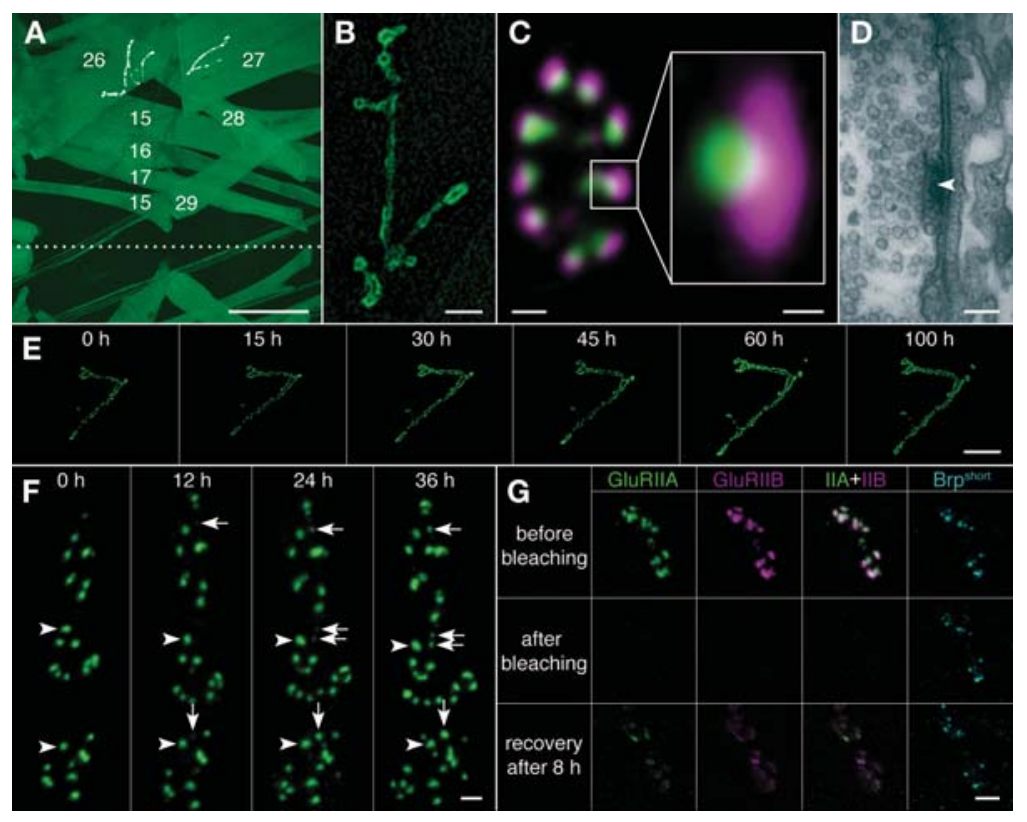

FIGURE 1. Intravital imaging of neuromuscular junctions (NMJs). (A) Selected body wall muscle fibers in an abdominal segment as seen from the exterior (outside-out view). The dotted line indicates the ventral midline. The image was modified to highlight NMJs at muscles 26 (VA1) and 27 (VA2). Construct used for the visualization of muscle fibers and NMJs: uas-dlgS97-gfp, expressed using c57-gal4. Scale bar, $100 \mu \mathrm{m}$. (B) Morphological structure of a Drosophila larval NMJ at muscle 27 (VA2), outlined by the expression of uas-dlgS97-gfp, using c57-gal4 as a driver. Scale bar, $10 \mu \mathrm{m}$. (C) Immunohistochemical stainings of a bouton of a larval NMJ and an individual synapse in lateral view (right box). Green, monoclonal antibody Bruchpilot ${ }^{\mathrm{Nc} 82}$ (Kittel et al. 2006); magenta, antibody against the glutamate receptor subunit DGluRIID (Qin et al. 2005). Scale bar (bouton), $1 \mu \mathrm{m}$; scale bar (synapse), $100 \mathrm{~nm}$. (D) Ultrastructure of an active zone. The arrowhead points at the T-bar, synaptic vesicles cluster next to it. Scale bar, $100 \mathrm{~nm}$. $(E, F)$ In vivo imaging of synapse formation at an identified NMJ at muscle 27, tracked using the GFP-labeled glutamate receptor subunit DGluRIIA. (Adapted, with permission, from Rasse et al. 2005.) (E) The development of an identified NMJ at muscle 27 tracked $>100 \mathrm{~h}$ at $16^{\circ} \mathrm{C}$. Scale bar, $10 \mu \mathrm{m}$. $(F)$ Higher magnification of the image shown in $E$ : New synapses form de novo (arrows). Mature synapses (arrowheads) remain stable. Scale bar, $2 \mu \mathrm{m}$. (G) In vivo fluorescent

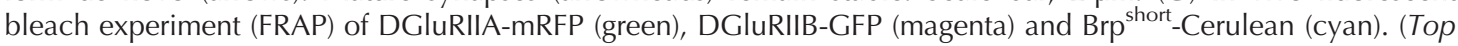
row) Images taken directly before bleaching. (Middle row) Images taken directly after bleaching of DGluRIIA and DGluRIIB. (Bottom row) Images taken $8 \mathrm{~h}$ after bleaching. Recovery of both DGluRIIA and DGluRIIB can be observed. Scale bar, $2 \mu \mathrm{m}$. 
and DiAntonio 2007). Moreover, similar to central synapses of mammals, synapses at Drosophila NMJs are not static structures but, rather, undergo activity-dependent and experience-dependent changes regarding the number of individual boutons and the overall number of synapses per NMJ (Zhong et al. 1992; Sigrist et al. 2003; Schmid et al. 2008).

Initial in vivo experiments of developing NMJs revealed that individual synapses (consisting of the presynaptic active zone [AZ] and the respective adjunct postsynaptic density [PSD]) assemble on a timescale of several hours, with pre- and postsynaptic proteins joining into the assembly of synapses in a temporally defined order (Rasse et al. 2005; Schmid et al. 2008; Fouquet et al. 2009). Clearly, it is intrinsically problematic to use exclusively static methods like immunohistochemistry when trying to reveal dynamic aspects of synapse assembly. This is particularly true because the assembly processes are not synchronized between individual synapses. Instead, the NMJ synapse population is a mosaic in terms of the age and maturation status of individual synapses.

Therefore, the temporal sequence of synaptic assembly and, moreover, turnover rates of proteins and axonal transport phenomena are questions that have to be addressed with approaches that make it feasible to track individually identified situations (e.g., distinct identified synapses or cargo containing vesicles within axons) over extended periods in vivo. Because the growth of individual synapses takes place over hours (Rasse et al. 2005; Schmid et al. 2008) and days, invasive methods that result in an immediate halt in developmental processes (e.g., opening the cuticle of a larva to image with waterimmersion lenses) are also problematic. With noninvasive in vivo imaging, on the other hand, larvae can be anesthetized for a limited amount of time and subsequently woken and fully recovered within minutes. Consequently, they can be imaged repeatedly during their life span as they develop (Rasse et al. 2005; Schmid et al. 2008; Fouquet et al. 2009) (Fig. 1E-G). The development of individual synapses as well as the history of particular proteins can readily be tracked and reconstructed on a molecular level.

\section{KEY PLAYERS IN SYNAPTIC ASSEMBLY AND DEVELOPMENT}

Each synapse consists of a presynaptic AZ (the site of transmitter release), a postsynaptic density, and a synaptic cleft separating them. The AZ is defined as the presynaptic region in which SVs fuse with the presynaptic membrane and release their cargo into the synaptic cleft. It can also be regarded as the area where pre- and postsynaptic membranes lie in planar opposition. The AZ can be further subdivided into the protein-rich cytomatrix at the active zone (CAZ), formed by several specialized proteins that play a role as a scaffold for other proteins (Zhai and Bellen 2004; Schoch and Gundelfinger 2006), and the associated machinery for the SV exocycle and endocycle (Siksou et al. 2007). In addition, a cytoplasmic pool of SVs is associated with the AZ. In the following paragraphs, several key proteins in synaptic development and function will be introduced and, if available, constructs suitable for in vivo imaging will be mentioned (these constructs are also listed in Table 1).

The fusion of SVs with the presynaptic membrane takes place on calcium influx through voltagegated calcium channels. A marker suitable for in vivo imaging of calcium channels is Cac1-green fluorescent protein (GFP) (Kawasaki et al. 2004). A very prominent structure of Drosophila AZs is an electron-dense projection called T-bar (Fig. 1D). The protein Bruchpilot, a member of the Cast/ERC/ ELKS family (Ohtsuka et al. 2002), is required for T-bar formation (Kittel et al. 2006). The monoclonal antibody Nc82, which labels Bruchpilot, serves as a canonical marker for AZs in Drosophila (Kittel et al. 2006; Wagh et al. 2006). Fluorescent fusion proteins of Bruchpilot are also available, which allow for the identification of AZs in vivo (Wagh et al. 2006; Schmid et al. 2008; Fouquet et al. 2009) (Fig. 1G). Transgenically expressed full-length Bruchpilot localizes to AZs but also aggregates at other sites. We found a labeled fragment of the protein $\left(\mathrm{Brp}^{\text {short }}\right.$, corresponding to amino acids 4731226 of the 1740-amino-acid Bruchpilot protein) that is especially well suited to identify AZs in vivo because it does not aggregate inappropriately. This fragment colocalizes with endogenous Bruchpilot and requires endogenous full-length Bruchpilot for its localization to AZs (Schmid et al. 2008; Fouquet et al. 2009). 
T.F.M. Andlauer and S.J. Sigrist

TABLE 1. Selected constructs suitable for in vivo imaging

\begin{tabular}{|c|c|c|c|}
\hline Protein & Fluorophore & Localization & Reference(s) \\
\hline Atrial natriuretic peptide (ANP) & GFP/Emerald & $\begin{array}{l}\text { Neuropeptidergic } \\
\text { vesicles }\end{array}$ & Rao et al. (2001) \\
\hline Bruchpilot & CFP, GFP, mStrawberry & Active zone & $\begin{array}{l}\text { Wagh et al. (2006), Schmid et al. } \\
\text { (2008), Fouquet et al. (2009) }\end{array}$ \\
\hline Calcium channel Cacophony (Cac1) & GFP & Active zone & $\begin{array}{l}\text { Kawasaki et al. (2004), Rasse et al. } \\
\text { (2005), Fouquet et al. (2009) }\end{array}$ \\
\hline Cytochrome c oxidase & GFP & Mitochondria & Pilling et al. (2006) \\
\hline Discs large (DlgS97) & GFP & $\begin{array}{l}\text { Subsynaptic reticulum } \\
\text { and muscle fibers }\end{array}$ & $\begin{array}{l}\text { Bachmann et al. (2004), Rasse et al. } \\
\text { (2005) }\end{array}$ \\
\hline DLiprin-a & GFP & Active zone & Fouquet et al. (2009) \\
\hline Fasciclin II & GFP & Periactive zone & Zito et al. (1999), Rasse et al. (2005) \\
\hline Glutamate receptor subunit DGluRIIA & GFP, mRFP & Postsynaptic density & $\begin{array}{l}\text { Rasse et al. (2005), Schmid et al. } \\
\quad(2008) \text {, Fouquet et al. (2009) }\end{array}$ \\
\hline Glutamate receptor subunit DGluRIIB & GFP, mRFP & Postsynaptic density & Schmid et al. (2008) \\
\hline Glutamate receptor subunit DGluRIIE & GFP & Postsynaptic density & Qin et al. (2005) \\
\hline PAK-kinase & GFP & Postsynaptic density & Rasse et al. (2005) \\
\hline Potassium channel construct CD8-Shaker & GFP & Subsynaptic reticulum & Zito et al. (1999), Rasse et al. (2005) \\
\hline Synaptobrevin & GFP & Synaptic vesicles & Zhang et al. (2002) \\
\hline Synaptotagmin & GFP & Synaptic vesicles & Zhang et al. (2002) \\
\hline
\end{tabular}

For a table of additional constructs, see Füger et al. (2007).

An additional protein associated with the $\mathrm{AZ}$ is DLiprin- $\alpha$, which interacts with several other $\mathrm{AZ}$ proteins (Kaufmann et al. 2002) and is also relevant for the transport of synaptic cargo (Miller et al. 2005). Recently, it was shown using in vivo imaging that DLiprin- $\alpha$ is incorporated into nascent synapses several hours before the arrival of Bruchpilot (Fouquet et al. 2009).

The presynaptic protein Syd-1 and the RIM family of proteins are known to play important roles in synapse development and function in other organisms (Owald and Sigrist 2009). To date, their role in Drosophila remains unclear. Bassoon and Piccolo, important components of the CAZ in mammals (Schoch and Gundelfinger 2006), have no homologs in Drosophila. Accordingly, an unknown combination of other proteins must take over their scaffolding role in the fly, with Bruchpilot being a primary candidate.

After the arrival of DLiprin- $\alpha$ at AZs, but before the incorporation of presynaptic Bruchpilot, ionotropic glutamate receptors accumulate at the postsynaptic site (Fouquet et al. 2009). Transgenic lines derived from genomic clones carrying fusions of fluorescent proteins with the receptor subunits DGluRIIA (dominant at immature synapses), DGluRIIB (incorporated during the maturation of synapses), and DGluRIIE (essential subunit of all DGluR receptors) are available. These fusion proteins have been shown to be functional at genetic, immunochemical, and electrophysiological levels (Qin et al. 2005; Rasse et al. 2005; Schmid et al. 2008) (Fig. 1E-G).

Drosophila larval NMJs have a simple architecture, which makes it possible to re-identify single synapses during successive imaging time points. This situation provides the opportunity to monitor subtle changes over time (e.g., the number of synapses, or the amount and distribution of a certain protein). It also means that individual synapses with a size of merely a few hundred nanometers can be re-identified during subsequent imaging sessions and, thus, protein trafficking and diffusion dynamics can be reconstructed. But to achieve this level of resolution, even the slightest movements of the larva have to be prevented throughout the imaging session.

The technique we describe in In Vivo Imaging of the Drosophila Larval Neuromuscular Junction (Andlauer and Sigrist 2012a) allows for the examination of live, intact larvae with a confocal microscope. Typically, larvae are imaged at several points in time during larval development, with the 
larvae waking up and roaming freely in between imaging time points. In this way, the normal, physiological development of identified synapses over extended periods of time can be studied in an intact animal. A protocol is also available for Building an Imaging Chamber for In Vivo Imaging of Drosophila Larvae (Andlauer and Sigrist 2012b). Analysis of the imaging data produced is described in Quantitative Analysis of Drosophila Larval Neuromuscular Junction Morphology (Andlauer and Sigrist 2012c).

To ensure high optical accessibility of the NMJs during the imaging procedure, the larvae are embedded in halocarbon oil, which has a refractive index very similar to their waxy cuticle. Thus, little reflection occurs on the interface between oil and cuticle, with the cuticle of the larvae becoming virtually transparent. The most superficial NMJs (at muscle fibers 26 [VA1] and 27 [VA2]; Fig. 1A) are the easiest to investigate as they lie directly below the cuticle, and no further light is lost by dispersion while passing through other tissues. However, deeper lying structures such as axon bundles can also be easily investigated.

The proteins of interest are labeled with a fluorescent marker tag (optimally monomeric fluorescent proteins to avoid aggregation [Shaner et al. 2005]). These proteins can then be expressed either under the control of their endogenous promoter at physiological expression levels (Rasse et al. 2005; Venken et al. 2008) or as overexpression constructs by using the flexibility of the GAL4-UAS system (Brand and Perrimon 1993). Furthermore, Drosophila allows for the evaluation of the functionality of the tagged proteins by rescue assays.

Although photobleaching can be problematic for weakly expressed proteins, the described imaging method is sensitive enough to typically image the same synapses several times or to observe the trafficking of proteins in time-lapse recordings.

The assay presented in In Vivo Imaging of the Drosophila Larval Neuromuscular Junction (Andlauer and Sigrist 2012a) is not restricted to the analysis of AZ assembly but can be applied to every field of interest in developing larvae that allows for reasonable optical access. For example, axonal transport is observed using markers for SVs (synaptobrevin-GFP and synaptotagmin-GFP [Zhang et al. 2002]), for neuropeptidergic vesicles (ANF-GFP) (Rao et al. 2001), or for mitochondria (Mito-GFP) (Pilling et al. 2006).

For a beginner who tries to orient in the "confusing jungle" of larval muscles and NMJs, starting with the observation of the expression of the protein Discs large (Dlg) with a postsynaptic driver (e.g., C57-GAL4 or MHC-GAL4) is recommended. Dlg is a major scaffolding component at larval NMJs and is mainly associated with the postsynaptic, complexly folded muscle membrane ("subsynaptic reticulum"). Two isoforms exist, DlgA, important for synapse development in embryonic stages, and DlgS97, the predominant form in adult flies (Mendoza-Topaz et al. 2008). Both proteins regulate AZ structure by influencing AZ length and spacing (Guan et al. 1996; Mendoza-Topaz et al. 2008). GFPtagged constructs of Dlg have been created (Bachmann et al. 2004) and applied for in vivo imaging (Rasse et al. 2005). Dlg overexpression in muscles outlines NMJs brightly and also stains muscle fibers at a more basal level (Fig. 1A,B) (Bachmann et al. 2004).

Table 1 lists a selection of constructs suitable for in vivo imaging. For additional constructs, see Füger et al. (2007). Also helpful is the GFP protein trap database (FlyTrap) derived from exon trap screens (http://flytrap.med.yale.edu).

Our assay is also amenable to study protein turnover and diffusion dynamics by FRAP (fluorescence recovery after photobleaching), preferably with a second, unbleached channel as a control (Fig. 1G), and by photoconversion (e.g., by using a protein tagged with the fluorescent marker protein Eos [Wiedenmann et al. 2004]). For protocols concerning FRAP, please consult previously published articles (Füger et al. 2007; Schmid and Sigrist 2008; Schmid et al. 2008).

This work was supported by grants from the Deutsche Forschungsgemeinschaft to S.J.S. (Exc 257, SI849/2-1 and 2-2, TP A16/SFB 551, TP B23/SFB581). We would like to thank Wernher Fouquet, 
Omid Khorramshahi, and Carolin Wichmann for contributions to Figure 1, as well as Richard W. Cho, Phillip A. Vanlandingham, and members of the Sigrist laboratory for critical comments on the manuscript. In addition, we would like to thank Gunther Tietsch (MSZ, University of Würzburg) for technical assistance regarding the construction of the imaging chamber and the anesthetization device.

\section{REFERENCES}

Andlauer TFM, Sigrist SJ. 2012a. In vivo imaging of the Drosophila larval neuromuscular junction. Cold Spring Harb Protoc doi: 10.1101/pdb. prot068593.

Andlauer TFM, Sigrist SJ. 2012b. Building an imaging chamber for in vivo imaging of Drosophila larvae. Cold Spring Harb Protoc doi: 10.1101/pdb. prot068585.

Andlauer TFM, Sigrist SJ. 2012c. Quantitative analysis of Drosophila larval neuromuscular junction morphology. Cold Spring Harb Protoc doi: 10.1101/pdb.prot068601.

Bachmann A, Timmer M, Sierralta J, Pietrini G, Gundelfinger ED, Knust E, Thomas U. 2004. Cell type-specific recruitment of Drosophila Lin-7 to distinct MAGUK-based protein complexes defines novel roles for Sdt and Dlg-S97. J Cell Sci 117: 1899-1909.

Brand AH, Perrimon N. 1993. Targeted gene expression as a means of altering cell fates and generating dominant phenotypes. Development 118: 401-415.

Broadie KS, Richmond JE. 2002. Establishing and sculpting the synapse in Drosophila and C. elegans. Curr Opin Neurobiol 12: 491-498.

Collins CA, DiAntonio A. 2007. Synaptic development: Insights from Drosophila. Curr Opin Neurobiol 17: 35-42.

C Crossley CA. 1978. The morphology and development of the Drosophila muscular system. in The genetics and biology of Drosophila (ed. Ashburner M, Wright T), pp. 499-560. Academic Press, New York.

Featherstone DE, Broadie K. 2000. Surprises from Drosophila: Genetic mechanisms of synaptic development and plasticity. Brain Res Bull 53: 501-511.

Fouquet W, Owald D, Wichmann C, Mertel S, Depner H, Dyba M, Hallermann S, Kittel RJ, Eimer S, Sigrist SJ. 2009. Maturation of active zone assembly by Drosophila Bruchpilot. J Cell Biol 186: 129145.

Füger P, Behrends LB, Mertel S, Sigrist SJ, Rasse TM. 2007. Live imaging of synapse development and measuring protein dynamics using two-color fluorescence recovery after photo-bleaching at Drosophila synapses. Nat Protoc 2: 3285-3298.

Garner CC, Waites CL, Ziv NE. 2006. Synapse development: Still looking for the forest, still lost in the trees. Cell Tissue Res 326: 249-262.

Guan B, Hartmann B, Kho YH, Gorczyca M, Budnik V. 1996. The Drosophila tumor suppressor gene, $d l g$, is involved in structural plasticity at a glutamatergic synapse. Curr Biol 6: 695-706.

Kaufmann N, DeProto J, Ranjan R, Wan H, Van Vactor D. 2002. Drosophila Liprin- $\alpha$ and the receptor phosphatase Dlar control synapse morphogenesis. Neuron 34: 27-38.

Kawasaki F, Zou B, Xu X, Ordway RW. 2004. Active zone localization of presynaptic calcium channels encoded by the cacophony locus of Drosophila. J Neurosci 24: 282-285.

Keshishian H, Broadie K, Chiba A, Bate M. 1996. The Drosophila neuromuscular junction: A model system for studying synaptic development and function. Annu Rev Neurosci 19: 545-575.

Kittel RJ, Wichmann C, Rasse TM, Fouquet W, Schmidt M, Schmid A, Wagh DA, Pawlu C, Kellner RR, Willig KI, et al. 2006. Bruchpilot promotes active zone assembly, $\mathrm{Ca}^{2+}$ channel clustering, and vesicle release. Science 312: 1051-1054.

Landgraf M, Thor S. 2006. Development and structure of motoneurons. Int Rev Neurobiol 75: 33-53.

Landgraf M, Sánchez-Soriano N, Technau GM, Urban J, Prokop A. 2003. Charting the Drosophila neuropile: A strategy for the standardised characterisation of genetically amenable neurites. Dev Biol 260: 207-225.

Mendoza-Topaz C, Urra F, Barría R, Albornoz V, Ugalde D, Thomas U, Gundelfinger ED, Delgado R, Kukuljan M, Sanxaridis PD, et al. 2008. DLGS97/SAP97 is developmentally upregulated and is required for complex adult behaviors and synapse morphology and function. J Neurosci 28: 304-314.

Miller KE, DeProto J, Kaufmann N, Patel BN, Duckworth A, Van Vactor D. 2005. Direct observation demonstrates that Liprin- $\alpha$ is required for trafficking of synaptic vesicles. Curr Biol 15: 684-689.

Nägerl UV, Köstinge G, Anderson JC, Martin KA, Bonhoeffer T. 2007. Protracted synaptogenesis after activity-dependent spinogenesis in hippocampal neurons. J Neurosci 27: 8149-8156.

Ohtsuka T, Takao-Rikitsu E, Inoue E, Inoue M, Takeuchi M, Matsubara K, Deguchi-Tawarada M, Satoh K, Morimoto K, Nakanishi H, et al. 2002. CAST: A novel protein of the cytomatrix at the active zone of synapses that forms a ternary complex with RIM1 and Munc13-1. J Cell Biol 158: 577-590.

Owald D, Sigrist SJ. 2009. Assembling the presynaptic active zone. Curr Opin Neurobiol 19: 311-318.

Pilling AD, Horiuchi D, Lively CM, Saxton WM. 2006. Kinesin-1 and dynein are the primary motors for fast transport of mitochondria in Drosophila motor axons. Mol Biol Cell 17: 2057-2068.

Prokop A. 2006. Organization of the efferent system and structure of neuromuscular junctions in Drosophila. Int Rev Neurobiol 75: 71-90.

Prokop A, Meinertzhagen IA. 2006. Development and structure of synaptic contacts in Drosophila. Semin Cell Dev Biol 17: 20-30.

Qin G, Schwarz T, Kittel RJ, Schmid A, Rasse TM, Kappei D, Ponimaskin E, Heckmann M, Sigrist SJ. 2005. Four different subunits are essential for expressing the synaptic glutamate receptor at neuromuscular junctions of Drosophila. J Neurosci 25: 3209-3218.

Rao S, Lang C, Levitan ES, Deitcher DL. 2001. Visualization of neuropeptide expression, transport, and exocytosis in Drosophila melanogaster. J Neurobiol 49: 159-172.

Rasse TM, Fouquet W, Schmid A, Kittel RJ, Mertel S, Sigrist CB, Schmidt M, Guzman A, Merino C, Qin G, et al. 2005. Glutamate receptor dynamics organizing synapse formation in vivo. Nat Neurosci 8: 898-905.

Schmid A, Sigrist SJ. 2008. Analysis of neuromuscular junctions: Histology and in vivo imaging. Methods Mol Biol 420: 239-251.

Schmid A, Hallermann S, Kittel RJ, Khorramshahi O, Frölich AM, Quentin C, Rasse TM, Mertel S, Heckmann M, Sigrist SJ. 2008. Activity-dependent site-specific changes of glutamate receptor composition in vivo. Nat Neurosci 11: 659-666.

Schoch S, Gundelfinger ED. 2006. Molecular organization of the presynaptic active zone. Cell Tissue Res 326: 379-391.

Schuster CM, Davis GW, Fetter RD, Goodman CS. 1996. Genetic dissection of structural and functional components of synaptic plasticity. I. Fasciclin II controls synaptic stabilization and growth. Neuron 17: 641-654.

Shaner NC, Steinbach PA, Tsien RY. 2005. A guide to choosing fluorescent proteins. Nat Methods 2: 905-909.

Sigrist SJ, Reiff DF, Thiel PR, Steinert J, Schuster CM. 2003. Experiencedependent strengthening of Drosophila neuromuscular junctions. $J$ Neurosci 23: 6546-6556.

Siksou L, Rostaing P, Lechaire JP, Boudier T, Ohtsuka T, Fejtová A, Kao HT, Greengard P, Gundelfinger ED, Triller A, et al. 2007. Three-dimensional architecture of presynaptic terminal cytomatrix. J Neurosci 27: 6868-6877.

Venken KJ, Kasprowicz J, Kuenen S, Yan J, Hassan BA, Verstreken P. 2008. Recombineering-mediated tagging of Drosophila genomic constructs for in vivo localization and acute protein inactivation. Nucleic Acids Res 36: e114.

Wagh DA, Rasse TM, Asan E, Hofbauer A, Schwenkert I, Dürrbeck H, Buchner S, Dabauvalle MC, Schmidt M, Qin G, et al. 2006. Bruchpilot, a protein with homology to ELKS/CAST, is required for structural 
integrity and function of synaptic active zones in Drosophila. Neuron 49: 833-844.

Wiedenmann J, Ivanchenko S, Oswald F, Schmitt F, Röcker C, Salih A, Spindler KD, Nienhaus GU. 2004. EosFP, a fluorescent marker protein with UV-inducible green-to-red fluorescence conversion. Proc Natl Acad Sci 101: 15905-15910.

Zhai RG, Bellen HJ. 2004. The architecture of the active zone in the presynaptic nerve terminal. Physiology (Bethesda) 19: 262-270.
Zhang YQ, Rodesch CK, Broadie K. 2002. Living synaptic vesicle marker: Synaptotagmin-GFP. Genesis 34: 142-145.

Zhong Y, Budnik V, Wu CF. 1992. Synaptic plasticity in Drosophila memory and hyperexcitable mutants: Role of cAMP cascade. J Neurosci 12: 644-651.

Zito K, Parnas D, Fetter RD, Isacoff EY, Goodman CS. 1999. Watching a synapse grow: Noninvasive confocal imaging of synaptic growth in Drosophila. Neuron 22: 719-729. 


\section{In Vivo Imaging of Drosophila Larval Neuromuscular Junctions to Study Synapse Assembly}

Till F.M. Andlauer and Stephan J. Sigrist

Cold Spring Harb Protoc; doi: 10.1101/pdb.top068577

\begin{tabular}{cc}
$\begin{array}{c}\text { Email Alerting } \\
\text { Service }\end{array}$ & Receive free email alerts when new articles cite this article - click here. \\
\hline $\begin{array}{c}\text { Subject } \\
\text { Categories }\end{array}$ & $\begin{array}{c}\text { Browse articles on similar topics from Cold Spring Harbor Protocols. } \\
\text { Confocal Microscopy (114 articles) } \\
\text { Developmental Biology (728 articles) } \\
\text { Drosophila (272 articles) } \\
\text { Imaging Development (255 articles) } \\
\text { Imaging for Neuroscience (342 articles) } \\
\text { In Vivo Imaging (334 articles) }\end{array}$ \\
\hline
\end{tabular}

